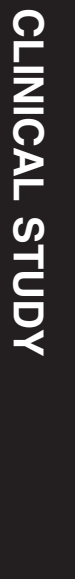

\title{
Plasma and vitreous fluid levels of Dickkopf-1 in patients with diabetic retinopathy
}

${ }^{1}$ Eye Institute of Xiamen University, Xiamen, China

${ }^{2}$ Eye Institute, Li Ka Shing Faculty of Medicine, University of Hong Kong, Hong Kong, China

${ }^{3}$ Xiamen Eye Center Affiliated to Xiamen University, Xiamen, China

${ }^{4}$ Department of Physiology, University of Oklahoma Health Sciences Center, Oklahoma City, OK, USA

Correspondence: J-x Ma, Department of Physiology, University of Oklahoma Health Sciences Center, Harold Hamm Diabetes Center, 941 Stanton L. Young Boulevard, BSEB 328B, Oklahoma City, OK 73104-5019, USA Tel: +1 405271 4372; Fax: +1 4052713973 . E-mail: jian-xing-ma@ ouhsc.edu or Z Liu, Eye Institute of Xiamen University, 168 Daxue Road, Xiamen, Fujian 361005, China Tel: +86 592 2186901; Fax: +86 5922186901 E-mail: zuguoliu@ xmu.edu.cn

Received: 25 February 2013 Accepted in revised form: 5 September 2013 Published online: 10 January 2014

\begin{abstract}
Purpose Dickkopf-1 (DKK-1) is a secreted inhibitor of the Wnt/ $\beta$-catenin signaling pathway, which plays a pathogenic role in diabetic retinopathy (DR). We aimed to investigate whether DKK-1 levels in the plasma and the vitreous are associated with DR in type 2 diabetes mellitus (DM) patients.
\end{abstract}

Methods Case-control study: plasma samples were collected from 125 type $2 \mathrm{DM}$ including 81 DR (29 non-proliferative DR (NPDR) and 52 proliferative DR (PDR)), 44 non-DR patients (NDR), and 100 non-diabetic controls. Undiluted vitreous fluid samples were obtained from 30 PDR and 25 nondiabetic patients. DKK-1 concentrations in samples were determined using enzymelinked immunosorbent assay. Variables were compared with the Kruskal-Wallis $\mathbf{H}$ test, Mann-Whitney U-test, and $\chi^{2}$-test, when appropriate.

Results Plasma DKK-1 levels were significantly lower in DR patients (median: $465.77 \mathrm{pg} / \mathrm{ml}$, range: $137.11-1190.31$ ) than in non-diabetic controls $(656.83 \mathrm{pg} / \mathrm{ml}, 171.63-$ 1795.08; $P<0.001)$ and NDR patients (693.04 pg/ml, 305.43-1218.35; $P<0.001$ ). Furthermore, DKK-1 levels were lower in PDR patients (425.21 pg/ml, 137.10-1077.32) compared with NPDR patients $(594.86 \mathrm{pg} / \mathrm{ml}$, 256.36-1393.27; $P=0.003)$. Vitreous absolute DKK-1 levels in PDR patients $(259.04 \mathrm{gg} / \mathrm{ml}$, 104.44-596.96) were higher than in nondiabetic controls (138.26 pg/ml, 18.69-239.52; $P<0.001)$. After normalizing by total vitreous protein concentrations, however, there was no significant difference between the groups. DKK-1 levels in vitreous were lower than those in plasma in both groups $(P<0.001$ for controls; $P=0.002$ for $P D R$ patients). Conclusions Decreased plasma DKK-1 levels, which may contribute to the Wnt

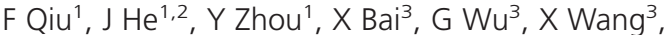
Z Liu' ${ }^{1}, Y$ Chen ${ }^{1}$, J-x Ma ${ }^{4}$ and Z Liu ${ }^{1}$ pathway activation, are associated with the presence and progression of DR, and have potential to become a biomarker for DR. Eye (2014) 28, 402-409; doi:10.1038/eye.2013.229; published online 10 January 2014

Keywords: diabetic retinopathy; Dickkopf-1; plasma; vitreous

\section{Introduction}

Diabetic retinopathy (DR) is one of the most common microvascular complications of diabetes mellitus (DM), which is a major cause of vision loss and blindness in working age populations, and its prevalence exhibits continuous increase. ${ }^{1}$ It is estimated that DR affects more than 10000 individuals per year. ${ }^{1}$ Nevertheless, there is no effective non-invasive treatment, because the pathogenesis of DR remains largely enigmatic. It is, therefore, necessary to identify the etiopathogenesis or the risk factor(s) associated with DR, and to develop new therapeutic strategies for this disease.

Identification of systemic and local factors associated with DR can provide new insights relating to the pathogenesis of DR and consequently contribute to the development of early diagnosis and new therapeutic modalities. Recently, great progress has been achieved in identifying soluble factors in the circulation and vitreous fluid, such as TNF- $\alpha$, VEGF, and sICAM-1, which are associated with the development and progression of DR. ${ }^{2-8}$

A recent study demonstrated that the canonical Wnt signaling pathway is activated in the retina in humans with DR and in DR animal models, and it has a causative role in DR. ${ }^{9}$ The Wnt/ $\beta$-catenin signaling pathway is an important signaling pathway that regulates multiple biological and pathological processes 
including cell proliferation and differentiation, apoptosis, stem cell maintenance, angiogenesis, inflammation, fibrosis, and carcinogenesis. ${ }^{10}$ This signaling pathway is regulated by a number of natural inhibitors including Dickkopf-1 (DKK-1), a well-established specific inhibitor of the Wnt signaling pathway. ${ }^{11}$ DKK-1 is a secreted protein and readily detectable in the circulation. ${ }^{12-16}$ Therefore, it is hypothesized that DKK-1 levels in circulation or ocular fluid could be associated with the development and progression of DR.

To test this hypothesis, the present study measured DKK-1 levels in the plasma and vitreous fluid from diabetic patients with and without DR, and compared them with those in non-diabetic controls.

\section{Materials and methods}

\section{Study participants}

This case-control study was conducted in Xiamen Eye Center Affiliated to Xiamen University from December 2009 to November 2010, and received approval from the Human Ethics committee of the center before this study, in accordance with the Declaration of Helsinki. Informed consent was obtained from each subject after the participants were informed with the purpose of the study and potential adverse effects of these procedures in detail.

Plasma samples were collected from consecutive type 2 diabetic patients with (DR) or without DR (NDR), and non-diabetic controls. All patients developed diabetes after the age of 30 years and were treated with insulin or oral anti-diabetic agents and an appropriate diet. Non-diabetic controls were age-matched individuals with no evidence of diabetes or ocular hemorrhages, exudation, or neovascularization who visited the center for routine examination, prescription of eyeglasses, or registering for cataract surgery during the same time frame. All subjects were of Han Chinese origin.

Exclusion criteria for ocular included previous intraocular surgery, intravitreal therapy, glaucoma, a history of ocular inflammation such as uveitis, and other ocular neovascular diseases such as retinal vein occlusion and age-related macular degeneration. Systemic exclusion criteria included renal dysfunction, hepatic dysfunction, ischemic cerebrovascular disorders, ischemic cardiovascular disorder, hematological diseases, connective tissue and systemic inflammatory diseases and history of malignancy, bone diseases, neurological disorder, and anti-platelet therapy, which may affect DKK-1 levels in circulation or ocular tissues.

In selected subjects, vitreous fluid samples were collected from proliferative DR (PDR) patients undergoing vitrectomy. Non-diabetic patients without ocular neovascularization and inflammation served as controls. All vitreous samples from patients with recent vitreous hemorrhage (within past 2 months) or in whom hemoglobin was detected in the vitreous were excluded.

All participants underwent clinical evaluation and a comprehensive ophthalmologic examination including best-corrected visual acuity, intraocular pressure evaluation, slit-lamp biomicroscopy, and dilated binocular ophthalmoscopy and B-scan ultrasound. Fundus photography (FP), fluorescein angiography (FA), or optical coherence tomography (OCT) was performed in DR patients.

All diagnoses of type 2 diabetes were based on criteria recommended by the World Health Organization. The diagnosis of retinopathy was made by an independent ophthalmologist using ophthalmoscopy, FP, FA, or OCT. Based on the international clinical DR severity scale, ${ }^{17}$ retinopathy was classified as no diabetic retinopathy (NDR), non-proliferative diabetic retinopathy (NPDR), or proliferative diabetic retinopathy (PDR). Participants were categorized according to the findings in the worse eye. Fasting blood glucose (FBG) and hemoglobin A1c (HbA1c) were reported by clinical laboratory. Hypertension was defined as a resting arterial blood pressure $\geq 140 / 90 \mathrm{~mm} \mathrm{Hg}$ or a history of antihypertensive drug therapy. Height and weight were measured to determine body mass index. Prior histories and personal characteristic habits and duration of diabetes were obtained from all participants via a questionnaire.

\section{Collection of blood samples and vitreous fluid samples}

Blood samples, drawn from the antecubital vein, were collected into tubes containing heparin. Following the centrifugation of the samples at $3000 \mathrm{RPM}$ for $10 \mathrm{~min}$ at $4{ }^{\circ} \mathrm{C}$, the plasma was separated and stored at $-80^{\circ} \mathrm{C}$ within $2 \mathrm{~h}$ until the assay was performed.

Undiluted vitreous fluid samples $(\sim 0.5 \mathrm{ml})$ from one eye per patient were collected through the vitreous cutter using a syringe attached to an automated vitrector before opening the infusion line at the start of a standard threeport pars plana vitrectomy. Samples were transferred to a tube, placed immediately on ice and centrifuged at 12000 r.p.m. for $5 \mathrm{~min}$ at $4{ }^{\circ} \mathrm{C}$. The supernatant was separated and stored at $-80^{\circ} \mathrm{C}$.

\section{Measurement of DKK-1 levels}

DKK-1 levels in the plasma and vitreous fluid were measured using a commercial enzyme-linked immunosorbent assay (ELISA) kits (R\&D Systems, Minneapolis, MN, USA). It is a sandwich enzyme immunoassay that permits DKK-1 measurements within 
a linear range of $31.3-2000 \mathrm{pg} / \mathrm{ml}$ with a detection limit of $15.6 \mathrm{pg} / \mathrm{ml}$. The assay allows sensitive and specific analysis of plasma DKK-1. The procedures were performed according to the instructions from the manufacturer. The laboratory personnel who performed ELISA were blind to the source of the samples, which were identified by numeric codes. All of the measurements were performed in triplicate for each sample, and the mean values were calculated. Inter- and intra-assay variations were $4.1 \%$ and $6.0 \%$, respectively.

\section{Measurement of vitreous hemoglobin}

To exclude those in whom intravitreous hemoglobin was detected, vitreous hemoglobin levels were determined by spectrophotometry (Uvikon 860, Kontron Instruments, Zurich, Switzerland), using the classic method of Harboe for measuring plasma hemoglobin in micromolar concentrations. ${ }^{18}$

\section{Measurement of total vitreous proteins}

Quantification of total proteins levels of centrifuged vitreous was performed by bicinchonic acid (BCA) colorimetric quantification (BCA Protein Assay Reagent kit; Pierce, Rockford, IL, USA) according to the manufacturer's instructions.

\section{Statistical analysis}

Statistical analyses were performed with SPSS (16.0; SPSS Inc., Chicago, IL, USA). Data were presented as the mean $\pm S D$, frequency (percentage), or median (range). Distribution of data was assessed using the KolmogorovSmirnov test. Depending on the distribution of data, independent sample $t$-tests or Mann-Whitney U-test was applied when only two comparisons were assigned. Kruskal-Wallis $\mathrm{H}$ test or one-way ANOVA was used for multiple comparisons. Chi-square test or Fisher's exact test was carried out to compare categorical variables. Comparisons were also made for the geometric mean plasma DKK-1 levels on a logarithmic scale between groups, after adjusting for age, gender, body mass index, current smoking (vs current nonsmoking), and hypertension (yes/no). A two-tailed $P$-value $<0.05$ was considered to indicate statistical significance.

\section{Results}

\section{Subjects' demographics}

The clinical characteristics of the subjects were summarized in Table 1. Plasma samples were collected from 125 type 2 diabetic patients, including $81 \mathrm{DR}$ patients and 44 NDR patients, and 100 non-diabetic controls. The 81 DR group included 29 NPDR patients and 52 PDR patients. Age and gender were matched among DR, NDR and control groups. Hypertension was more common in DR and NDR groups than in controls, but its prevalence did not vary significantly in the DR and NDR. There was no significant difference in body mass index, smoking status, duration of DM, FBG, and $\mathrm{HbA} 1 \mathrm{c}$ levels between DR group and NDR group.

Vitreous fluid samples were harvested from eyes of 30 patients with PDR and 25 controls. In PDR, vitrectomy was performed for persistent vitreous hemorrhage with capillary neovascularization on the retinal surface $(n=12)$, tractional retinal detachment with the presence of fibrotic, regressed, retinal neovascularization $(n=13)$ or macular edema $(n=5)$. Controls were non-diabetic ocular diseases including patients with idiopathic macular holes $(n=12)$, rhegmatogenous retinal detachment patients $(n=8)$, or idiopathic epiretinal membrane patients $(n=5)$.

\section{Plasma levels of DKK-1 in DR patients}

There was a statistically significant difference of DKK-1 levels in the plasma among DR, NDR, and non-diabetic control groups ( $P<0.001$, Kruskal-Wallis $\mathrm{H}$ test). As shown in Figure 1a, the levels of DKK-1 were significantly lower in the DR group (median: 465.77 $\mathrm{pg} / \mathrm{ml}$, range: 137.11-1190.31) in comparison with those in non-diabetic controls $(656.83 \mathrm{pg} / \mathrm{ml}, 171.63-1795.08$; $P<0.001$, Mann-Whitney U-test) and NDR groups (693.04 pg/ml, 305.43-1218.35; $P<0.001$, Mann-Whitney U-test). Adjusted geometric mean (95\%CI) DKK-1 levels on a logarithmic scale remained lower in those with DR (457.40 (412.09-509.33) pg/ml), compared with either non-diabetic controls (659.17 (598.41-724.44) pg/ml; $P<0.001)$ or those with NDR (691.03 (599.79-794.33) $\mathrm{pg} / \mathrm{ml} ; \mathrm{P}<0.001)$, after adjustment for age, gender, smoking status, BMI, and hypertension. No statistical difference in DKK-1 levels was found between NDR patients and healthy controls $(P=0.571$, Mann-Whitney U-test). These findings demonstrated that decreased DKK-1 levels in the circulation are associated with the presence or development of DR. In addition, we further analyzed the relation of DKK-1 levels to the severity of DR. DKK-1 levels in the plasma were lower in PDR patients $(425.21 \mathrm{pg} / \mathrm{ml}, 137.10-1077.32)$ than in NPDR patients (594.86 pg/ml, 256.36-1393.27; $P=0.003$, Mann-Whitney U-test; Figure 1b). Adjusted geometric mean $(95 \% \mathrm{CI}) \mathrm{DKK}-1$ levels remained lower in those with PDR (408.31 (358.92-464.52) pg/ml), compared with NPDR patients (561.04 (472.06-665.27) pg/ml; $P=0.022$ ), after adjustment for age, gender, smoking status, BMI, 
Table 1 Demographics and clinical characteristics of study subjects ${ }^{\mathrm{a}}$

\begin{tabular}{|c|c|c|c|c|c|}
\hline \multirow[t]{2}{*}{ Characteristic } & \multicolumn{3}{|c|}{ Plasma samples } & \multicolumn{2}{|c|}{ Vitreous fluid } \\
\hline & $\begin{array}{l}\text { DR group } \\
(\mathrm{n}=81)\end{array}$ & $\begin{array}{l}\text { NDR group } \\
\quad(\mathrm{n}=44)\end{array}$ & $\begin{array}{l}\text { Control group } \\
(\mathrm{n}=100)\end{array}$ & $\begin{array}{l}\text { PDR group } \\
\quad(\mathrm{n}=30)\end{array}$ & $\begin{array}{c}\text { Control group } \\
(\mathrm{n}=25)\end{array}$ \\
\hline $\operatorname{Sex}(\mathrm{F} / \mathrm{M} ; n)$ & $43 / 38$ & $24 / 20$ & $56 / 44$ & $16 / 14$ & $12 / 13$ \\
\hline Age (years) & $62(36-83)$ & $64(35-83)$ & $66(37-84)$ & $56(43-63)$ & $56(36-68)$ \\
\hline Hypertension, $n(\%)$ & $38(46.9)^{\mathrm{b}}$ & $19(47.5)^{\mathrm{b}}$ & $22(22.0)$ & $13(43.3)$ & $9(39.1)$ \\
\hline Current smokers, $n(\%)$ & $11(13.6)$ & 7 (15.9) & $12(12.0)$ & $5(16.7)$ & $3(12.0)$ \\
\hline Body mass index, $\left(\mathrm{kg} / \mathrm{m}^{2}\right)$ & $27.91 \pm 3.00$ & $28.09 \pm 3.67$ & $27.22 \pm 2.83$ & $28.08 \pm 3.12$ & $27.52 \pm 2.95$ \\
\hline Duration of DM (years) & $9(2-30)$ & $6(1-20)$ & - & $10(2-30)$ & - \\
\hline $\mathrm{FBG}(\mathrm{mmol} / \mathrm{l})$ & $7.44 \pm 2.27$ & $7.26 \pm 2.13$ & - & $7.27 \pm 2.19$ & - \\
\hline $\operatorname{HbA1c}(\%)$ & $8.65 \pm 1.35$ & $8.97 \pm 1.68$ & - & $8.78 \pm 1.43$ & - \\
\hline \multirow[t]{3}{*}{ Retinal pathologies $(n)$} & NPDR (29) & - & - & VH (12) & IMH (12) \\
\hline & PDR (52) & & & TRD (13) & RRD (8) \\
\hline & & & & ME (5) & IEM (5) \\
\hline
\end{tabular}

Abbreviations: DR, diabetic retinopathy; FBG, free blood glucose; IEM, idiopathic epiretinal membrane; IMH, idiopathic macular holes; ME, macular edema; NDR, diabetic patients without DR; PDR, proliferative DR; RRD, rhegmaogenous retinal detachment; TRD, tractional retinal detachment; $\mathrm{VH}$, vitreous hemorrhage.

The chi-square test was carried out to compare categorical variables (sex, smoking status, and hypertension); Kruskal-Wallis test was used for multiple comparisons of age; Mann-Whitney U-test was applied when only two comparisons of age, duration of DM; one-way ANOVA was performed to compare body mass index among DR, NDR and control groups; independent sample $t$-tests was applied for comparisons of FBG or HbA1c between NDR and DR groups.

${ }^{a}$ Data were expressed as number (\%), mean $\pm \mathrm{SD}$, or median (range).

$\mathrm{b}_{\text {vs }}$ control, $P<0.05$.
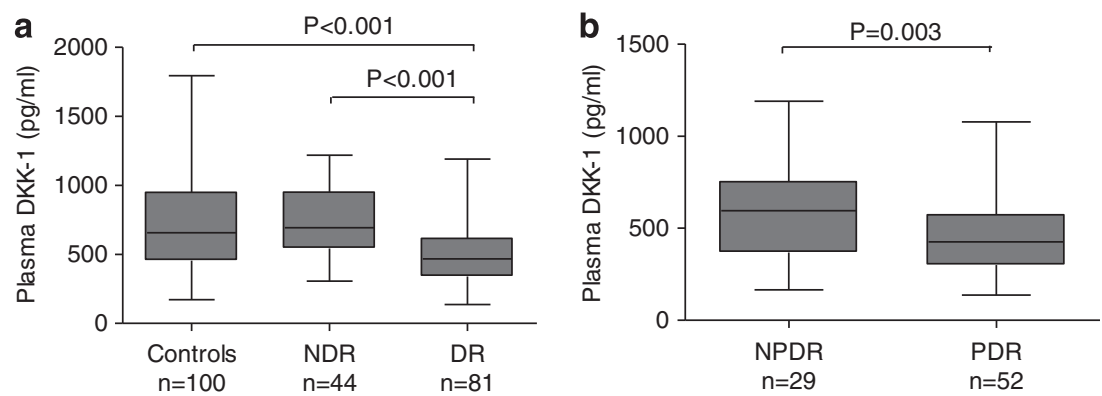

Figure 1 Decreased plasma DKK-1 levels in DR patients. (a) Comparison of DKK-1 concentrations in the plasma of non-diabetic individuals (controls), diabetic patients without retinopathy (NDR), and diabetic retinopathy (DR). (b) Comparison of KK-1 levels between patients with non-proliferative diabetic retinopathy (NPDR) and patients with proliferative diabetic retinopathy (PDR). Data were analyzed with Mann-Whitney U-test. The boxes represent the 25th and 75th percentile (the lower and upper quartiles, respectively), and the band near the middle of the box represents the 50th percentile (the median). The ends of the whiskers represent the minimum and maximum.

and hypertension. These findings revealed that decreased DKK-1 levels in the circulation are associated with severity or progression of DR.

\section{Vitreous fluid concentrations of DKK-1}

The absolute concentrations of vitreous DKK-1 levels were significantly higher in the patients with PDR (259.04 pg/ml, 104.44-596.96) than in controls with nondiabetic ocular diseases (138.26 pg/ml, 18.69-239.52;

$P<0.001$, Mann-Whitney U-test; Figure 2a). To determine the influence of the breakdown of the bloodretina barrier (BRB) and, in consequence, the increased blood diffusion that occurs in diabetic patients with PDR, the vitreous DKK-1 concentrations were normalized for total vitreous protein concentration. We detected higher intravitreous protein concentrations in diabetic patients with PDR $(3.03 \mathrm{mg} / \mathrm{ml}, 0.59-7.06)$ than in non-diabetic patients $(0.99 \mathrm{mg} / \mathrm{ml}, 0.36-1.96 ; P<0.001$; Figure $2 \mathrm{~b})$. After adjusting for total vitreous protein concentration, DKK-1 levels (ratio of DKK-1/total vitreous protein concentrations) in patients with PDR $(118.24 \mathrm{pg} / \mathrm{mg}$, 61.02-177.02) were similar to those in the control group (122.21 pg/mg, 51.90-199.03; $P=0.710$; Figure 2c).

\section{Vitreous fluid vs plasma concentrations of DKK-1}

DKK-1 levels were significantly lower in the vitreous fluid than in the plasma from both controls $(P<0.001$; Figure $3 a)$ and PDR patients $(P=0.002$; Figure $3 b)$. In 

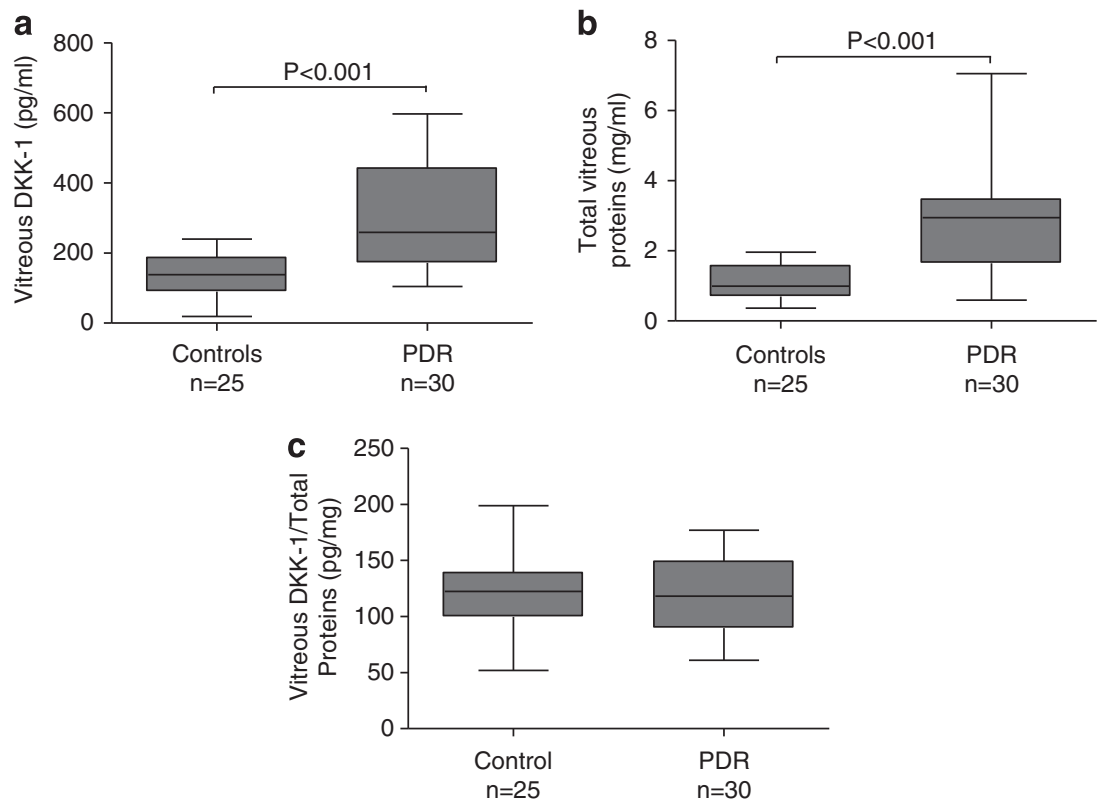

Figure 2 DKK-1 levels in the vitreous of PDR. (a) Comparison of DKK-1 concentrations between proliferative diabetic retinopathy (PDR) patients and controls. (b) Comparison of total vitreous proteins concentrations between PDR patients and controls. (c) Comparison of DKK-1 levels (ratio of DKK-1 to total vitreous proteins (pg/mg)) after adjusting for the total vitreous proteins between PDR patients and controls. Controls were non-diabetic patients with other retinal pathologies consisting of idiopathic macular holes patients $(n=12)$, hegmaogenous retinal detachment $(n=8)$, idiopathic epiretinal membrane $(n=5)$. Data were analyzed with Mann-Whitney U-test. The boxes represent the 25th and 75th percentiles (the lower and upper quartiles, respectively), and the band near the middle of the box represents the 50th percentiles (the median). The ends of the whiskers represent the minimum and maximum.
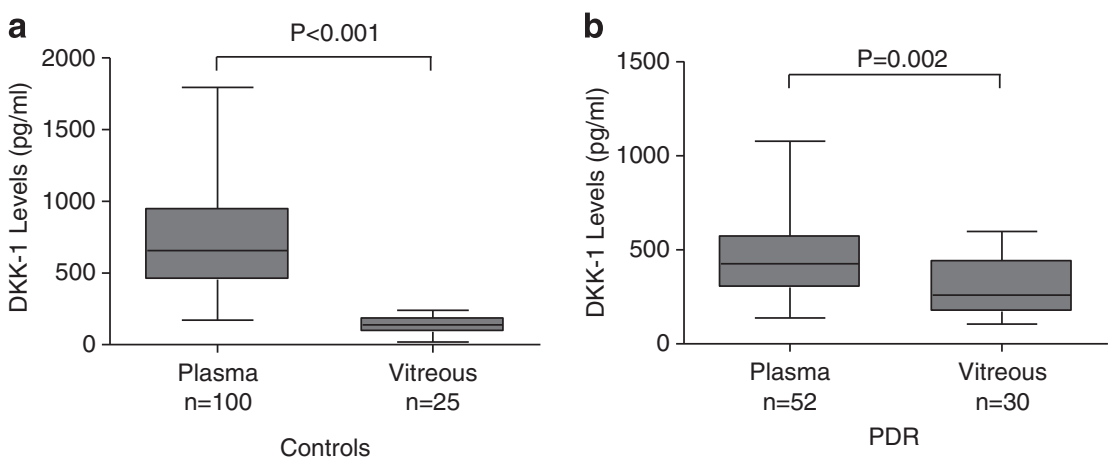

Figure 3 Comparison of DKK-1 levels in vitreous fluid and plasma. (a) Comparison of DKK-1 levels in vitreous and plasma of controls. (b) Comparison of DKK-1 levels in vitreous and plasma of PDR patients. Data were analyzed with Mann-Whitney U-test. The boxes represent the 25th and 75th percentiles (the lower and upper quartiles, respectively), and the band near the middle of the box represents the 50th percentiles (the median). The ends of the whiskers represent the minimum and maximum.

addition, it was noted that DKK-1 concentrations in the vitreous were not as high as those in the plasma in all subjects.

\section{Discussion}

To our best knowledge, this is the first report to measure levels of DKK-1 in circulation and human vitreous fluid of DR patients. It is reported that alterations of circulating DKK-1 protein levels have been shown to be associated with various diseases such as multiple myeloma, rheumatoid arthritis, breast cancer, lung cancer, and esophageal cancer patients. ${ }^{12-16}$ However, there is no documented study to associate circulating DKK-1 levels with DR. There is also no information available concerning the DKK-1 level in the vitreous fluid.

In our study, it was shown that DKK-1 levels in the plasma were significantly lower in patients with DR when compared with non-diabetic subjects and NDR patients. Furthermore, they were further lower in patients with PDR, which is an advanced stage of DR, 
compared with those in patients with NPDR. These results suggest that decreased DKK-1 levels in the circulation might be related to the presence and severity or progression of DR.

We do not know at present whether the change represents primary causes or consequences of DR. The nature of our study does not permit us to determine whether low levels of DKK-1 can accurately predict subsequent DR events. DKK-1 is an inhibitor of the Wnt signaling pathway and platelets are the major source of circulating DKK-1. ${ }^{19}$ It was demonstrated that the canonical Wnt signaling pathway has a causative role in DR in animal models. ${ }^{9}$ Taking into account such reports and our present results, it is speculated that some events in DM could influence the secretion of DKK-1 from the platelet, leading to decrease of DKK-1 levels, and subsequently, the decreased DKK-1 levels might contribute to the aberrant activation of the Wnt signaling pathway that could promote retinal inflammation and neovascularization in DR by the upregulation of inflammatory and proangiogenic factors including TNF- $\alpha$, ICAM- 1 , and VEGF. ${ }^{9,20}$ Thus, it seems reasonable to postulate that decreased DKK-1 levels may have an influence on the development and progression of DR and have the potential to become a biomarker for prediction of DR. Our study also suggests that medications to induce expression of DKK-1 or external supplement DKK-1 could be of value for preventing and treating DR. However, the therapeutic value of DKK-1 in the treatment of DR in humans requires further investigation. Further study would also be necessary to investigate factors affecting DKK-1 levels in circulation.

The vitreous fluid obtained from patients submitted to vitreoretinal surgery could be a useful material for indirectly exploring special factor levels in retina. In the present study, DKK-1 concentrations were found to be higher in the vitreous fluid in PDR patients when compared with those in the controls. However, apart from local production, the elevated proteins levels in the vitreous can be influenced by the blood levels through vitreous hemorrhage and the disruption of the bloodretinal barrier as found in DR. In this study, the influence of vitreous hemorrhage was ruled out, as all vitreous samples, in which recent clinical vitreous hemorrhage ( $<2$ months) was reported or hemoglobin was detected in the vitreous, were rejected. The breakdown of the blood-retinal barrier that exists in DR could facilitate the passage of DKK-1 from systemic circulation into the vitreous body. Our results revealed the increase of intravitreal total protein concentration observed in PDR patients in comparison with non-diabetic controls and no significant difference of DKK-1 concentrations between DR and non-diabetes groups was found after normalization to total vitreous proteins concentrations.
These findings suggest that the enhancement of DKK-1 in patients with PDR mainly derive from the blood diffusion due to the disruption of blood-retinal barrier but not local production in retina.

It is reported that the DKK-1 mRNA is expressed at low levels in most other normal human tissues, such as the heart, liver, lung, kidney, brain, and bone marrow. ${ }^{21}$ This result was supported by our data in this study that DKK-1 was at low levels (18.69-239.52 pg/ml) in human vitreous, being strikingly lower than those in the plasma in controls. Our result suggests that DKK-1 may be produced at low levels in ocular tissues, and little of the circulating DKK-1 may leak into the ocular fluid under normal conditions. In PDR patients, the vitreous levels of DKK-1, even the highest recorded here $(596.96 \mathrm{pg} / \mathrm{ml})$, was also lower than that in the plasma $(752.02 \mathrm{pg} / \mathrm{ml})$. These indicated that the increased DKK-1 level in the vitreous humor of PDR patients would not be sufficient to inhibit the abnormal activation of the Wnt signaling pathway, regardless of its origin, suggesting that subretinal or intravitreal injection of DKK-1 could be a therapeutic strategy for DR.

Our study has the following limitations. First, it is a case-control study and further longitudinal clinical studies are necessary to clarify whether or not lower circulating DKK-1 levels are more susceptible to DR or diabetic vascular complications, or measuring DKK-1 levels is a useful means to aid early diagnosis, prospectively determine prognosis. Second, the vitreous fluid obtained from patients undergoing vitreoretinal surgery is an indirect measure. However, it is very difficult to perform a direct method of quantification of DKK-1 levels in the human retina. Finally, as the number of study subjects was notlarge, we did not evaluate the relationship between DKK-1 levels and clinical characteristics such as gender, age, levels of FBG or $\mathrm{HbA1c}$, duration and therapy of diabetes, presence, duration and therapy of concomitant disease hypertension and polyneuropathy, which should be confirmed by further studies.

In conclusion, we first investigated DKK-1 levels in the circulation in DR patients, and provided the first evidence of DKK-1 presence in the vitreous fluid. Our results showed that decreased DKK-1 levels are associated with the presence and the progression of DR. The decreased circulating DKK-1 levels may contribute to the Wnt pathway activation in retina in DR, and have potential to become a biomarker for prediction of DR. DKK-1 was detected at very low levels in the vitreous humor. Our study may potentially have clinical importance, which will not only enhance understanding of the pathogenic mechanisms of DR, especially PDR, or ocular vascular disorders, but also could provide basis for new 
strategies for PDR treatment, or provide evidence to develop a novel biomarker for prediction and prognosis of this disease.

\section{Summary}

What was known before

- Canonical Wnt signaling pathway is activated in the retina in humans with DR and in DR animal models, and it has a causative role in DR.

- DKK-1, a secreted protein, is a specific inhibitor of the Wnt signaling pathway.

What this study adds

- We measured DKK-1 levels in the plasma and vitreous fluid from patients with DR.

- Plasma DKK-1 levels were significantly lower in DR patients than those in non-diabetic subjects and NDR patients. They were further lower in PDR patients compared with NPDR patients. These results suggest that decreased DKK-1 levels in the circulation might be related to the presence and severity or progression of DR.

- Vitreous absolute DKK-1 levels in PDR patients were higher than those in non-diabetic controls, due to the disruption of blood-retinal barrier but not local production in retina.

- DKK-1 was at low levels in human vitreous, being strikingly lower than those in the plasma in controls.

\section{Conflict of interest}

The authors declare no conflict of interest.

\section{Acknowledgements}

This research was supported by grants from the National Basic Research Program of China (Project973), grant no.2011CB504606 (Zuguo Liu); National Natural Science Foundation of China, Beijing, China, grant no. 30931160432, 30872810 (Zuguo Liu); Technological Innovation Platform Program of Fujian Province, China, grant no. 2009J1013 (Zuguo Liu); NIH EY019309 (J-xM); NIH EY018659(J-xM); Cross-strait Technological Platform of Chinese Medicine grant no. 3502Z20100006 (Zuguo Liu).

\section{Author contributions}

Design of the study (FQ, J-xM, Zuguo Liu); conduct of the study (FQ, JH, XB, XW, GW, Zhen Liu); analysis and interpretation of the data (FQ, YZ, YC, J-xM, Zuguo Liu); writing the paper $(F Q, Y Z)$; review and approval of the manuscript (J-xM, Zuguo Liu). Zuguo Liu and J-xM had full access to all the data in the study and take responsibility for the integrity of the data and the accuracy of the data analysis.

\section{References}

1 Fong DS, Aiello LP, Ferris 3rd FL, Klein R. Diabetic retinopathy. Diabetes Care 2004; 27: 2540-2453.

2 Patel JI, Saleh GM, Hykin PG, Gregor ZJ, Cree IA. Concentration of haemodynamic and inflammatory related cytokines in diabetic retinopathy. Eye 2008; 22: 223-228.

3 Doganay S, Evereklioglu C, Er H, Turkoz Y, Sevinc A, Mehmet $\mathrm{N}$ et al. Comparison of serum NO, TNF-alpha, IL-1beta, sIL-2R, IL-6 and IL-8 levels with grades of retinopathy in patients with diabetes mellitus. Eye 2002; 16: 163-170.

4 Malik RA, Li C, Aziz W, Olson JA, Vohra A, McHardy KC et al. Elevated plasma CD105 and vitreous VEGF levels in diabetic retinopathy. J Cell Mol Med 2005; 9: 692-697.

5 Burgos R, Mateo C, Canton A, Hernandez C, Mesa J, Simo R. Vitreous levels of IGF-I, IGF binding protein 1, and IGF binding protein 3 in proliferative diabetic retinopathy: a case-control study. Diabetes Care 2000; 23: 80-83.

6 Aiello LP, Avery RL, Arrigg PG. Vascular endothelial growth factor in ocular fluid of patients with diabetic retinopathy and other retinal disorders. New Engl J Med 1994; 331: 480-487.

7 Kado S, Nagata N. Circulating intercellular adhesion molecule-1, vascular cell adhesion molecule-1, and E-selectin in patients with type 2 diabetes mellitus. Diabetes Res Clin Pract 1999; 46: 143-148.

8 Maberley D, Cui JZ, Matsubara JA. Vitreous leptin levels in retinal disease. Eye 2006; 20: 801-804.

9 Chen Y, Hu Y, Zhou T, Zhou KK, Mott R, Wu M et al. Activation of the Wnt pathway plays a pathogenic role in diabetic retinopathy in humans and animal models. Am J Pathol 2009; 175: 2676-2685.

10 Wodarz A, Nusse R. Mechanisms of Wnt signaling in development. Annu Rev Cell Dev Biol 1998; 14: 59-88.

11 Zhang B, Ma JX. Wnt pathway antagonists and angiogenesis. Protein Cell 2010; 1: 898-906.

12 Kaiser M, Mieth M, Liebisch P, Oberlander R, Rademacher J, Jakob C et al. Serum concentrations of DKK-1 correlate with the extent of bone disease in patients with multiple myeloma. Eur J Haematol 2008; 80: 490-494.

13 Voorzanger-Rousselot N, Journe F, Doriath V, Body JJ, Garnero P. Assessment of circulating Dickkopf-1 with a new two-site immunoassay in healthy subjects and women with breast cancer and bone metastases. Calcif Tissue Int 2009; 84: 348-354.

14 Yamabuki T, Takano A, Hayama S, Ishikawa N, Kato T, Miyamoto $\mathrm{M}$ et al. Dikkopf-1as a novel serologic and prognostic biomarker for lung and esophageal carcinomas. Cancer Res 2007; 67: 2517-2525.

15 Lane NE, Nevitt MC, Lui LY, de Leon P, Corr M. Wnt signaling antagonists are potential prognostic biomarkers for the progression of radiographic hip osteoarthritis in elderly Caucasian women. Arthritis Rheum 2007; 56: 3319-3325.

16 Sheng SL, Huang G, Yu B, Qin WX. Clinical significance and prognostic value of serum Dickkopf-1 concentrations in patients with lung cancer. Clin Chem 2009; 55: 1656-1664.

17 Wilkinson CP, Ferris 3rd FL, Klein RE, Lee PP, Agardh CD, Davis $\mathrm{M}$ et al. Proposed international clinical diabetic retinopathy and diabetic macular edema disease severity scales. Ophthalmology 2003; 110: 1677-1682.

18 Fairbanks VF, Ziesmer SC, O'Brien PC. Methods for measuring plasma hemoglobin in micromolar concentration. Clin Chem 1992; 38: 132-140. 
19 Voorzanger-Rousselot N, Goehrig D, Facon T, Clezardin P, Garnero P. Platelet is a major contributor to circulating levels of Dickkopf-1: clinical implications in patients with multiple myeloma. Br J Haematol 2009; 145: 264-266.

20 Zhou T, Hu Y, Chen Y, Zhou KK, Zhang B, Gao G et al. The pathogenic role of the canonical Wnt pathway in age-related macular degeneration. Invest Ophthalmol Vis Sci 2010; 51: 4371-4379.

21 Forget MA, Turcotte S, Beauseigle D, Godin-Ethier J, Pelletier S, Martin J et al. The Wnt pathway regulator DKK1 is preferentially expressed in hormone-resistant breast tumours and in some common cancer types. $\mathrm{Br} \mathrm{J}$ Cancer 2007; 96: 646-653. 